\title{
Previsão dos Preços de Commodities por Meio das Taxas de Câmbio
}

\author{
Davi Rosolen \\ Pesquisador - Louis Dreyfus Commoditties \\ Endereço para contato: Av. Brigadeiro Faria Lima, 1355 - 140 andar - São Paulo - Brasil \\ CEP: 01452-919 - E-mail: davibond@hotmail.com
}

\author{
Michael Viriato Araújo \\ Professor - Insper Instituto de Ensino e Pesquisa \\ Endereço para contato: Rua Quatá, 300 - sala 710 - Vila Olímpia - São Paulo - Brasil \\ CEP: 04546-042 - E-mail: michael.viriato@insper.edu.br
}

\section{Marco Lyrio}

Professor - Insper Instituto de Ensino e Pesquisa

Endereço para contato: Rua Quatá, 300 - sala 706 - Vila Olímpia - São Paulo - Brasil

CEP: 04546-042 - E-mail: marco.lyrio@insper.edu.br

Recebido em 06 de fevereiro de 2012. Aceito em 07 de agosto de 2013.

\section{Resumo}

Este trabalho procura modelar e prever o comportamento dos preços de commodities utilizando taxas de câmbio de países exportadores de commodities. A compreensão do comportamento desses preços é importante para um apropriado controle da inflação e planejamento da produção. Os resultados obtidos apontam para uma relação de causalidade entre a taxa de câmbio e os preços de commodities para os países estudados, com exceção da África do Sul e Argentina. Para Austrália, Brasil, Canadá, Chile, Colômbia e Nova Zelândia, a taxa de câmbio se mostra uma informação significativa para previsões de preços de commodities para o período dentro da amostra. No caso da Austrália e do Canadá, a relação também é significativa para o período fora da amostra. Os resultados encontrados confirmam os obtidos por Chen, Rogoff e Rossi (2010), além de estender aquele trabalho aos casos da Argentina, Brasil e Colômbia.

\section{Palavras-Chave}

commodities, taxa de câmbio, teste de causalidade de Granger, modelo de previsão

\begin{abstract}
This paper aims to model and predict the behavior of commodity prices using the exchange rates of commodity-exporting countries. Understanding commodity price dynamics is important for a proper control of inflation and planning of production. Our results point to a causality relation between the exchange rate and commodity prices for all countries under study except South Africa and Argentina. For Australia, Brazil, Canada, Chile, Colombia and New Zealand the exchange rate is an important piece of information to forecast commodity prices in-sample. For Australia and Canada, this relation is also significant out-of-sample. Our results confirm those of Chen, Rogoff and Rossi (2010) and extend that work to the cases of Argentina, Brazil, and Colombia.
\end{abstract}




\section{Keywords}

commodities, exchange rate, Granger causality test, forecasting model

\section{JEL Classification}

F31, F37

\section{Introdução}

A procura por modelos com capacidade de prever movimentos de preços e variáveis econômicas é recorrente na literatura de finanças. Este trabalho se insere nesse contexto, visto que tem como objetivo explicar e prever a variação no preço das commodities utilizando taxas de câmbio. A literatura nesse sentido é relativamente recente e, de certa forma, é fruto de uma literatura correlata, já bem mais explorada, que diz respeito à elaboração de modelos de previsão da taxa de câmbio. ${ }^{1}$ Apesar dos avanços alcançados nessa área, ainda se buscam modelos que sejam capazes de prever a taxa de câmbio com relativa precisão dentro e fora da amostra e para diferentes moedas e momentos econômicos. Isso pode ser comprovado pela dificuldade que esses modelos têm em superar um simples passeio aleatório, conforme observado por Meese e Rogoff (1983).

Recentemente, Engel e West (2005) sugeriram uma possível explicação para esse fato. Esses autores utilizaram um modelo de valor presente com expectativas racionais ${ }^{2}$ para mostrar que o comportamento teórico da taxa de câmbio pode ser, na verdade, muito parecido com um passeio aleatório. Isso é o caso quando o modelo utilizado tem como base fundamentos macroeconômicos com raízes unitárias e inclui, também, um fator de desconto próximo de $1,{ }^{3}$ características defendidas pelos autores como razoáveis. A conclusão desse raciocínio é que os fundamentos macroeconômicos não têm poder de previsão sobre as taxas de câmbio. Os autores ressaltam, contudo, que as taxas de câmbio podem, ao invés disso, ser utilizadas para prever tais fundamentos econômicos. Isso se deve ao fato de que boa parte da variação de curto prazo nas taxas de câmbio pode ser explicada por mudanças nas expectativas com relação aos valores

1 Ver Sarno e Valente (2009) e as referências listadas neste artigo.

2 A seguinte equação ilustra tal modelo: $s_{t}=(1-b) \sum_{j=0}^{\infty} b^{j} E_{t}\left(a_{1}^{\prime} x_{t+j}\right)+b \sum_{j=0}^{\infty} b^{j} E_{t}\left(a_{2}^{\prime} x_{t+j}\right), 0<b<1$, em que $\mathrm{s}_{t}$ é o preço de um ativo (por exemplo, o logaritmo da taxa de câmbio), $x_{t}$ é um vetor em $R^{n}$ de fundamentos macroeconômicos, $b$ é um fator de desconto, e $a_{1}$ e $a_{2}$ e são vetores em $R^{n}$.

3 Quanto mais o fator de desconto for próximo de 1 , maior será o peso hoje dos fundamentos futuros. 
futuros de tais fundamentos. Dessa forma, a taxa de câmbio passa a ser útil para a previsão desses fundamentos econômicos, sendo sugerida a existência de causalidade no sentido de Granger com relação aos mesmos.

Este arcabouço teórico forneceu o alicerce para novos horizontes de pesquisa na medida em que sugeriu uma forte correlação entre alterações nos fundamentos futuros e inovações nas taxas de câmbio. Entretanto, os testes empíricos feitos por Engel e West (2005) com fundamentos macroeconômicos tais como oferta e demanda de moeda, taxa de juros, choque de produtividade, entre outros, não se mostraram consistentes. $\mathrm{O}$ modelo de cointegração bivariado não obteve resultados satisfatórios para a cointegração entre fundamentos e taxas de câmbio, devido provavelmente a problemas de endogeneidade. Este problema pode ser minimizado, por sua vez, quando o escopo de estudo são as commodity currencies e o fator macroeconômico em questão é o preço das commodities ponderado pela pauta de exportações destes países. Conforme definido em Chen, Rogoff e Rossi (2010), ${ }^{4}$ o termo acima utilizado é atribuído às moedas que possuem relação com preços de commodities, pois seus países possuem uma pauta de exportação concentrada em commodities.

Este trabalho se insere em contexto no qual os fundamentos que são modelados e previstos por taxas de câmbio são os preços de commodities. Para isto, nós primeiramente testamos a causalidade de Granger entre as variáveis e posteriormente analisamos o poder de previsão de uma variável sobre a outra. O enfoque é a capacidade de previsão do preço das commodities por meio das taxas de câmbio, replicando o estudo proposto por CRR (2010) e extendendo-o a mais três casos, o argentino, o brasileiro e o colombiano. $\mathrm{O}$ estudo desses países se justifica por também apresentarem características dos exportadores de commodities analisados em CRR (2010), ou seja, países nos quais a receita com essas exportações representa $50 \%$ ou mais da receita total de exportação.

O fenômeno enigmático que consiste na tendência de movimento conjunto dos preços de commodities distintas foi estudado por Pindyck e Rotemberg (1990). Esses autores mostram que variáveis macroeconômicas, tais como inflação, demanda agregada, taxas de juros e câmbio podem explicar a correlação e padrões de variação

4 A partir desse ponto, denominaremos o trabalho de Chen, Rogoff e Rossi (2010) como CRR (2010). 
de preços de diferentes commodities. ${ }^{5}$ As mudanças destas variáveis macroeconômicas afetam a oferta e demanda de boa parte das commodities globais, impactando diretamente nos preços. Esta relação ocorre de duas maneiras, sendo a primeira de forma direta, com alteração da oferta e demanda de commodities. Um exemplo para este caso é uma redução da taxa de juros da economia, que aumenta a demanda agregada e, portanto, a demanda por petróleo e cobre para a produção industrial, ao mesmo tempo em que o aumento da renda eleva a demanda por cacau e trigo (commodities não industriais). A segunda maneira se dá de forma indireta através da mudança de expectativas. Neste caso, alterações nas variáveis macroeconômicas afetam a expectativa sobre a oferta e demanda futura de commodities, uma vez que estas são bens estocáveis, e a mudança nas expectativas altera a demanda por estoques e, consequentemente, os preços correntes.

A análise de Pindyck e Rotemberg (1990) teve como base sete commodities escolhidas de forma a serem as mais não correlacionadas possíveis. Trigo, algodão, cobre, ouro, petróleo, madeira serrada e cacau são produzidas em diferentes regiões, com influências climáticas distintas, têm diferentes usos, algumas produzidas através da agricultura e outras extraídas da natureza.

Trata-se de um grupo bastante heterogêneo, sendo que elas não são substituíveis entre si ou produzidas de forma conjunta. Portanto, eliminando o componente macroeconômico dos preços dessas commodities, estes se tornariam não correlacionados. Ou seja, espera-se que os preços dessas commodities se movam de forma conjunta exclusivamente em resposta à percepção dos agentes com relação aos fundamentos econômicos. ${ }^{6}$

\footnotetext{
5 As variáveis explicativas selecionadas e testadas no trabalho em referência foram: inflação, produção industrial, títulos do governo americano (T-Bill de 3 meses), taxa de câmbio (cesta de moedas igualmente ponderada composta por Libras Esterlinas, Marco Alemão e Yen), base monetária (M1), e índice de ações S\&P. Na estimação com um lag, todas as variáveis são significantes ao nível de um porcento, com exceção do índice de ações S\&P. Com seis lags, todas as variáveis são significantes ao nível de um porcento, com exceção da produção industrial, significante ao nível de cinco porcento.

6 Após estimar a influência dos fundamentos econômicos e eliminar o seu efeito sobre o movimento de preços das commodities, verificou-se que a correlação de preços permaneceu devido à limitação estatística do modelo utilizado. Foi utilizado, consequentemente, um modelo de variáveis latentes, o qual minimizou o problema, mas não corrigiu o enigma do comovimento entre os preços das commodities analisadas.
} 
Neste sentido, o escopo do presente trabalho não se limita a analisar a influência dos fatores macroeconômicos, mas também dimensionar a importância desta influência para o caso das taxas de câmbio.

Com isso, justifica-se a utilização da taxa de câmbio de países exportadores de commodities (commodity currency) para modelar o preço desses produtos. A escolha desta variável se deve à sua característica "forward looking". Conforme CRR (2010), a taxa de câmbio nominal responde às expectativas sobre mudanças futuras nos fundamentos econômicos e, por isto, pode ajudar a prevê-los. O contrário, ou seja, a utilização de fundamentos como preço de commodities para a previsão de taxa de câmbio, não apresenta resultados empíricos consistentes.

$\mathrm{Na}$ verdade, não é novidade na literatura corrente a ideia de que variações nas taxas de câmbio de países exportadores de commodities estão correlacionadas ao movimento de preços das commodities. Entretanto, CRR (2010) evidencia uma relação de causalidade sistemática entre estas variáveis, além de elaborarem modelos com poder de previsão fora da amostra. Esses autores mostram que flutuações nas taxas de câmbio de países como África do Sul, Austrália, Canadá, Chile e Nova Zelândia apresentam poder de previsão sobre o futuro dos preços das commodities globais. ${ }^{7}$

Seguindo o modelo de CRR (2010), elabora-se aqui um índice de commodities ponderado pela pauta de exportação de cada país em estudo com a finalidade de identificar a relação de causalidade e analisar as propriedades de previsão da taxa de câmbio sobre este índice. Isso é feito através de testes de causalidade de Granger e de uma modelagem econométrica para previsão dentro e fora da amostra. Também são realizados testes para se comparar a acurácia entre os diferentes modelos.

Os principais resultados deste trabalho podem ser sintetizados em três pontos. Primeiro, a taxa de câmbio causa o índice de preços de commodities no sentido de Granger - e não o contrário para Austrália, Brasil, Canadá, Chile, Colômbia e Nova Zelândia. Segundo, para esses países, a previsão do movimento de preços de

7 Testes de causalidade de Granger robustos a instabilidades e previsões fora da amostra mostraram que taxas de câmbio Granger-causam preços das commodities para cada país estudado. Foi evidenciado, também, que a combinação de "commodity currencies" analisadas pode ajudar na previsão dos movimentos do mercado global de commodities. 
commodities utilizando a taxa de câmbio se mostra eficiente para previsões dentro da amostra, quando comparado ao modelo de passeio aleatório. Terceiro, quando o mesmo modelo é aplicado fora da amostra os resultados não são tão satisfatórios. Apesar do modelo apresentar um menor erro de previsão comparado ao passeio aleatório, não é possível comprovar significância estatística para esta diferença.

O restante do trabalho é dividido da seguinte forma. Na seção seguinte, serão descritas a base de dados com as variáveis a serem analisadas e a metodologia utilizada. Na terceira seção serão detalhadas as ferramentas econométricas, bem como serão testadas as séries e desenvolvidos os modelos de previsão para o preço das commodities. As conclusões do trabalho são apresentas na última seção.

\section{Base de Dados e Metodologia}

Segundo a metodologia do Fundo Monetário Internacional (FMI), ${ }^{8}$ há um amplo conjunto de países que podem ter suas moedas classificadas como commodity currencies, o que abre um leque de possibilidades de amostragem para trabalhos como este, que se propõe a estudar o comportamento de commodity currencies como fatores para previsão do preço de commodities. Todavia, limita-se aqui o escopo a algumas moedas e países que se destacam nos trabalhos correlatos a este, além de incluir alguns países exportadores importantes na América Latina, tais como Brasil, Argentina e Colômbia.

Austrália, Canadá e Nova Zelândia são países industrializados com economias voltadas para a exportação de commodities. África do Sul e Chile são países em desenvolvimento que também têm forte peso das commodities em suas exportações. Dada a importância de commodities para essas economias, a pequena proporção das mesmas frente à economia global e o longo horizonte temporal de taxas de câmbio flutuantes, esses países são frequentemente utilizados para analisar a relação entre commodities e taxa de câmbio e, por este

8 Utilizamos a classificação do FMI para commodity currencies de países em desenvolvimento, a qual foi utilizada por Cashin et al. (2004), que consideram a importância de commodities na pauta de exportações. Mais especificamente, se as receitas de exportação de commodities representarem $50 \%$ ou mais das receitas de exportações totais do país, a moeda em referência é considerada como uma commodity currency. Estendemos tal classificação para os seguintes países industrializados: Austrália, Canadá e Nova Zelândia. 
motivo, também foram considerados neste trabalho. ${ }^{9}$ A extensão do estudo para países da América Latina tem por motivo a importância crescente destas economias na dinâmica mundial. Selecionados os países, o passo seguinte é a construção de um índice de commodities para cada um deles, considerando o peso de suas exportações. Posteriormente, esse índice deve ser analisado em conjunto com as séries de preços das respectivas moedas.

\subsection{Base de Dados}

A coleta de dados foi feita para os preços de commodities, os pesos das principais commodities exportadas por cada país estudado e as taxas de câmbio com relação ao dólar americano. As séries temporais de preços de commodities são mensais e têm como fonte os dados do FMI e da Bloomberg.

Para cada país, as commodities exportadas foram ordenadas em ordem crescente de peso na pauta de exportações, sendo que apenas as primeiras commodities que atingiram um mínimo de 50\% da exportação total foram selecionadas. A partir dessa composição foi atribuído um peso a cada commodity, com soma total de $100 \%$, proporcional ao seu peso original na pauta de exportações de cada país individualmente.

Desta forma, os pesos das commodities exportadas para Austrália, Canadá e Nova Zelândia são diferentes dos utilizados em CRR (2010), que utilizaram um maior número de commodities na amostra. No caso da África do Sul e do Chile, os pesos foram os mesmos utilizados em CRR (2010). Para a Argentina e Colômbia, os pesos foram extraídos do trabalho de Cashin et al. (2004). ${ }^{10}$ Já para o Brasil, calculou-se o peso médio do valor mensal das principais commodities exportadas de janeiro de 2006 a julho de 2011. ${ }^{11}$ A Tabela 1 resume os pesos que formam os índices utilizados para cada país neste trabalho.

\footnotetext{
9 Mesmo que sejam os principais produtores de algumas das commodities globais, esses países são considerados como tomadores de preços no âmbito das análises realizadas.

10 Tabela Al, pág.246, Cashin et al. (2004).

11 Fonte: Ministério do Desenvolvimento, Indústria e Comércio (MDIC).
} 
Tabela 1 - Pesos utilizados para a composição do índice de preços de commodities

\begin{tabular}{|c|c|c|c|c|c|c|c|}
\hline \multicolumn{2}{|c|}{ África do Sul } & \multicolumn{2}{|l|}{ Argentina } & \multicolumn{2}{|c|}{ Austrália } & \multicolumn{2}{|l|}{ Brasil } \\
\hline OURO & $48,00 \%$ & FARELO DE SOJA & $42,86 \%$ & CARVÃO & $41,01 \%$ & MINÉRIO DE FERRO & $31,80 \%$ \\
\hline PLATINA & $30,00 \%$ & TRIGO & $30,95 \%$ & OURO & $15,80 \%$ & PETRÓLEO & $21,82 \%$ \\
\hline CARVÃO & $22,00 \%$ & MILHO & $26,19 \%$ & $\begin{array}{l}\text { MINÉRIO } \\
\text { DE FERRO } \\
\text { TRIGO } \\
\text { ALUMÍNIO }\end{array}$ & $\begin{array}{r}15,63 \% \\
13,95 \% \\
13,61 \%\end{array}$ & $\begin{array}{l}\text { SOJA EM GRÃO } \\
\text { AÇÚCAR } \\
\text { CARNE DE FRANGO } \\
\text { CAFÉ }\end{array}$ & $\begin{array}{r}18,12 \% \\
10,40 \% \\
9,62 \% \\
8,24 \%\end{array}$ \\
\hline Canadá & & Chile & & Colômbia & & Nova Zelândia & \\
\hline PETRÓLEO & $36,58 \%$ & COBRE & $100,00 \%$ & CAFÉ & $56,47 \%$ & CARNEIRO & $32,98 \%$ \\
\hline MADEIRA & $23,25 \%$ & & & CARVÃO & $22,35 \%$ & CARNE BOVINA & $24,80 \%$ \\
\hline GÁS NATURAL & $18,29 \%$ & & & BANANA & $21,18 \%$ & ALUMÍNIO & $21,90 \%$ \\
\hline CARNE BOVINA & $13,33 \%$ & & & & & $L \tilde{A}$ & $20,32 \%$ \\
\hline ALUMÍNIO & $8,55 \%$ & & & & & & \\
\hline
\end{tabular}

Diferentemente da periodicidade trimestral utilizada em CRR (2010), os dados utilizados na análise econométrica têm periodicidade mensal e a amostra, mais atualizada que em CRR (2010), conta com 360 observações entre agosto de 1981 e julho de 2011, no caso de países como Austrália, África do Sul, Canadá e Nova Zelândia. Para os demais países, a amostra foi limitada pelo histórico de preços das moedas, sendo considerados apenas períodos em que as taxas de câmbio tiveram flutuação.

O período compreendido na análise para o Chile foi de janeiro de 1998 a julho de 2011 com 163 observações. Diferentemente do período utilizado em CRR (2010), não foi considerado o período anterior a 1998, pois até 1997 o regime cambial chileno era baseado em bandas cambiais com elevada rigidez. Apenas a partir de 1998, este país aumentou a flexibilidade de sua moeda, com bandas de flutuação chegando a 16\% e tornando-se totalmente flexível em 1999. Para a Colômbia, foi considerado o mesmo período e número de observações. Para o Brasil, a amostra abrangeu o período de abril de 1999 a julho de 2011, ou seja, um período com 148 observações e subsequente ao overshooting cambial de 1999. No caso da Argentina, foi considerado o período posterior à maxidesvalorização do peso e início do regime de câmbio flutuante, ou seja, de julho de 2002 a julho de 2011, com 108 observações. 


\section{2 Índices de Preços de Commodities}

Os índices de preços de commodities (ic) compostos especificamente para cada país foram construídos com os pesos $(w)$ descritos na Tabela 1 acima e os preços do mercado mundial para as referidas commodities $(p)$. Para evitar um possível viés devido às diferenças no nível de preço das commodities, estes foram normalizados para a mesma base. Logo, o índice de preços ponderados geometricamente pelos valores normalizados $(p)$ de cada commodity na pauta de exportação dos países selecionados foi calculado da seguinte forma:

$$
i c_{i, t}=\left(\sum_{j=1}^{j} w_{j} p_{j, t}\right),
$$

onde $t$ representa o período, $i$ o país, $j$ a commodity em referência e $w_{j}$ o peso de cada item, dado pelo valor das exportações da commodity $j$ sobre o total do valor das principais commodities $j$ exportadas pelo país $i$.

Uma vez construídas as séries temporais para os índices de commodities, aplicou-se o teste de Dickey-Fuller Aumentado (ADF) de raízes unitárias. Com base nesse teste, não foi possível rejeitar a hipótese nula de que as séries ic continham raízes unitárias. A partir disto, passou-se a analisar as séries diferenciadas em primeira ordem. $\mathrm{O}$ mesmo teste foi aplicado para as séries do logaritmo das taxas de câmbio. Da mesma forma, não foi possível rejeitar a hipótese da existência de raízes unitárias e, por isso, estas séries também foram diferenciadas em primeira ordem.

\subsection{Métodos Econométricos}

Este trabalho tem como objetivo evidenciar a relação de causalidade entre as variáveis em estudo (taxas de câmbio e preços de commodities), bem como o poder preditivo de uma variável sobre a outra. Para a relação de causalidade, aplicou-se o teste de causalidade de Granger. Os modelos de previsão foram estimados através do método de mínimos quadrados ordinários com matriz de covariância de Newey-West, robusta a heterocedasticidade e autocorrelação. 
A estimação dos parâmetros dos modelos desconsiderou as últimas 12 observações de cada série, compreendidas aqui como fora da amostra.

Os testes de previsão de uma variável sobre a outra tiveram como referência o modelo de passeio aleatório. Depois de construídos e calibrados os parâmetros, os modelos preditivos foram testados dentro e fora da amostra. Para o teste dentro da amostra, foi considerado o período até julho de 2010 para cada país, dadas as séries descritas no item 2.l. Já para o teste fora da amostra, considerou-se o período de agosto de 2010 a julho de 2011, em um total de 12 observações. Posteriormente, foram comparadas as previsões do modelo proposto ao modelo de referência e aos últimos 12 meses observados na amostra para cada país. Para mensurar o poder de previsão, comparou-se o erro quadrático médio da previsão do modelo estimado ao do modelo de referência. Os resultados dos modelos também foram testados estatisticamente de acordo com os procedimentos de avaliação comparativa de acurácia de previsões propostos por Diebold e Mariano (1995). ${ }^{12}$ Esta análise foi realizada para o conjunto de observações consideradas dentro e fora da amostra de modo segregado. Os resultados obtidos com os testes são discutidos nas próximas seções.

\section{Resultados}

Nesta seção são abordados os dois temas centrais deste trabalho, a identificação da relação de causalidade entre o preço das commodities e a taxa de câmbio para os países estudados e a criação de um modelo de previsão a partir da relação de causalidade verificada.

\subsection{Relação de Causalidade entre as Variáveis}

É recorrente na literatura econômica relacionar movimentos nas taxas de câmbio dos países em desenvolvimento, cuja pauta de exportações está baseada em commodities primárias, com flutuações nos preços mundiais das commodities indicando estes como causa para aqueles (Cashin et al. (2004) e Veríssimo et al. (2012)).

\footnotetext{
12 Trata-se de um teste assintótico fundamentado na diferença da função de perda das séries de previsão, que tem por objetivo avaliar a acurácia das previsões comparadas. A hipótese nula é a de igual acurácia entre as previsões, que é equivalente a dizer que a média populacional do diferencial de perda das séries é igual a zero.
} 
A ideia geral para isto é que, ceteris paribus, um aumento no preço de uma determinada commodity resulta em um efeito renda ao país produtor, que recebe mais dólares por suas exportações e assim teria sua moeda nacional fortalecida. ${ }^{13}$ Entretanto, tais argumentos teóricos mostram-se pouco práticos para a literatura empírica fundamentada na modelagem de taxas de câmbio. Cashin et al. (2004) examinam este tema através de testes de cointegração, endogeneidade e causalidade, tendo como objetivo determinar quantos países exportadores de commodities têm suas taxas reais de câmbio explicadas por movimentos nos preços reais das commodities. Diferentemente de Cashin et al. (2004) e Veríssimo et al. (2012), não se intenciona verificar a existência de cointegração entre as variáveis taxa de câmbio e preço de commodities, mas determinar se existe uma relação de causalidade entre elas.

O método de investigação utilizado para evidenciar a causalidade entre as variáveis neste estudo foi o teste de causalidade de Granger, conforme CRR (2010). Considerando duas variáveis aleatórias $x$ e $y$, este teste pode ser realizado por meio da equação de regressão:

$$
E_{t}\left(\Delta y_{t+1}\right)=\beta_{0}+\beta_{1} \Delta x_{t}+\beta_{2} \Delta y_{t}
$$

onde $E_{t}\left(\Delta y_{t+1}\right)$ é o valor esperado de $\Delta y$ no tempo $t+1$. A causalidade de Granger, ou seja, se a variável $\Delta x_{t}$ causa $\Delta y_{t}$ no sentido de Granger, é verificada caso a hipótese nula $\beta_{1}=0$ seja rejeitada. Aplicando esta metodologia para o caso em questão, para cada país a relação de causalidade foi testada entre as variáveis índices de preços de commodities e logaritmo das taxas de câmbio, nesta ordem e na ordem inversa (taxas de câmbio causam índices de preços de commodities). Os resultados são apresentados na Tabela 2.

Tabela 2

\begin{tabular}{lllllllll}
\hline & AS & ARG & AUS & BR & CAN & CHI & COL & NZ \\
\hline P-valor & 0,3381 & $0,0726^{\star}$ & 0,4697 & 0,2106 & 0,6744 & 0,8318 & 0,3285 & $0,0032^{\star *}$ \\
Estatística F & 0,9199 & 3,2880 & 0,5237 & 1,5815 & 0,1767 & 0,0452 & 0,9605 & 8,7961 \\
\hline
\end{tabular}

Nota: AS, África do Sul; ARG, Argentina; AUS, Austrália; BR, Brasil; CAN, Canadá; CHI, Chile; COL, Colômbia; NZ, Nova Zelândia

* Rejeita-se H0 com nível de significância de 10\%, indicando evidência de causalidade de Granger.

** Rejeita-se H0 com nível de significância de 5\%, indicando evidência de causalidade de Granger.

${ }^{13}$ Outro possível canal de alteração dos termos de troca é o efeito Balassa-Samuelson, o qual uma alteração na produtividade do setor de bens comercializáveis altera os salários e preços relativos da economia e, por consequência, a taxa de câmbio real. 
Conforme se observa na Tabela 2, para um nível de significância de 10\%, o teste não indicou evidência de que o índice de preços de commodities causa a taxa de câmbio no sentido de Granger, exceto nos casos de Argentina e Nova Zelândia. Apesar desses resultados contradizerem o senso econômico explicitado anteriormente, vão de encontro aos resultados obtidos por CRR (2010). Esses autores não encontraram evidência desta causalidade para todos os países estudados, incluindo a Nova Zelândia.

Os argumentos propostos por Engel e West (2005) indicam que a relação inversa - ou seja, a taxa de câmbio causando os fundamentos econômicos no sentido de Granger - faz sentido, uma vez que variações de curto prazo nas taxas de câmbio podem ser explicadas por mudanças nas expectativas com relação aos fundamentos econômicos. No modelo apresentado por Engel e West (2005), a taxa de câmbio é uma função da esperança com relação aos fundamentos no futuro e do fator de desconto utilizado. CRR (2010) também argumentam neste sentido, destacando a importância da taxa de câmbio como variável precedente aos fundamentos econômicos. Esta relação pode ser representada por:

$$
s_{t}=\gamma \sum_{j=0}^{\infty} \varphi^{j} E_{t}\left(f_{t+1} \mid I_{t}\right),
$$

onde $\gamma$ e $\varphi$ são parâmetros dados pelas especificações do modelo e $E_{t}$ é a esperança estatística dada a informação $I_{t}$. Essa relação mostra que o logaritmo da taxa de câmbio $(s)$ deve Granger causar os seus fundamentos $(f)$. Para o âmbito deste trabalho, o índice de preços de commodities representa tais fundamentos econômicos. Como mostrado a seguir, encontram-se evidências empíricas em favor desta abordagem teórica. A análise desta proposição foi realizada através do teste de causalidade de Granger indicado na Tabela 3.

Tabela 3

\begin{tabular}{ccccccccc}
\hline & AS & ARG & AUS & BR & CAN & CHI & COL & NZ \\
\hline P-valor & 0,2765 & 0,1647 & $0,0000^{* *}$ & $0,0132^{* *}$ & $0,0933^{*}$ & $0,0008^{\star *}$ & $0,0054^{* *}$ & $0,0000^{* *}$ \\
Estatística F & 1,1878 & 1,9574 & 22,3137 & 6,2958 & 2,8308 & 11,5812 & 7,9436 & 27,5051 \\
\hline
\end{tabular}

Nota: AS, África do Sul; ARG, Argentina; AUS, Austrália; BR, Brasil; CAN, Canadá; CHI, Chile; COL, Colômbia; NZ, Nova Zelândia

* Rejeita-se H0 com nível de significância de 10\%, indicando evidência de causalidade de Granger.

** Rejeita-se H0 com nível de significância de 5\%, indicando evidência de causalidade de Granger. 
Conforme se observa na Tabela 3, a hipótese nula de que a taxa de câmbio não Granger causa o índice de preços de commodities é rejeitada para os países em análise, excetuando-se a África do Sul e Argentina, para um nível de significância de 10\%. Ou seja, há evidência de que a taxa de câmbio Granger causa o índice de preços de commodities para Austrália, Brasil, Canadá, Chile, Colômbia e Nova Zelândia. Este resultado confirma o obtido por CRR (2010). Entretanto, tendo em vista a diferença de períodos para a amostra, em CRR (2010) não foi encontrada evidência desta causalidade para a Austrália e Nova Zelândia, ao contrário da África do Sul.

A partir desses resultados, apenas para os países em que houve rejeição da hipótese nula, indicando causalidade de Granger para estas variáveis, justifica-se o desenvolvimento de modelos de previsão do índice de preços de commodities através da taxa de câmbio.

\subsection{Modelos de Previsão}

Uma vez verificada a relação de causalidade entre as variáveis, o próximo passo é a construção de modelos capazes de prever o índice de preços de commodities utilizando a taxa de câmbio, que é a variável Granger-causa. Para esta fase, os países nos quais não foram encontrados indícios de causalidade de Granger foram retirados da amostra, nesse caso a África do Sul e a Argentina. Para avaliar a eficiência destes modelos, definiu-se como referência o modelo de passeio aleatório, comparando as previsões dentro e fora da amostra.

O modelo proposto neste trabalho utiliza a variação da taxa de câmbio como indicador antecedente às variações nos índices de preços de commodities, além do componente autorregressivo. A especificação do modelo é dada por:

$$
E_{t} \Delta i c_{t+1}=\beta_{0 t}+\beta_{1 t} \Delta i c_{t}+\beta_{2 t} \Delta s_{t}
$$

onde $\Delta i c_{t} \mathrm{e} \Delta s_{t}$ são as primeiras diferenças do índice de preços de commodities e do logaritmo da taxa de câmbio, respectivamente, no período $t$. 
Este modelo, denominado Modelo Commodity Câmbio (MCC), foi aplicado para Austrália, Brasil, Canadá, Chile, Colômbia e Nova Zelândia, considerando o índice de preços de commodities e a taxa de câmbio de cada país. Em seguida, foram geradas as séries de previsão dentro da amostra e comparadas com um passeio aleatório, descrito por:

$$
\Delta i c_{t+1}=\mu+\Delta i c_{t}+\varepsilon_{t+1},
$$

onde $\mu$ representa a tendência do processo estocástico e $\varepsilon_{t+1}$ é uma sequência i.i.d. com média zero e variância $\sigma_{\varepsilon}^{2}$.

Clements e Hendry (1998) apresentam critérios para análises absolutistas sobre a acurácia de modelos de previsão. Entretanto, esses autores ressaltam os problemas relativos à utilidade destes critérios devido às peculiaridades intrínsecas de cada modelo. Como alternativa natural, a avaliação de previsões por comparação a modelos de referência é um método utilizado recorrentemente na literatura econométrica. Neste sentido, o critério de avaliação mais frequente é comparar o erro quadrático médio da previsão (EQMP) do modelo proposto versus do modelo de referência.

Comparou-se o EQMP do MCC ao EQMP do modelo de passeio aleatório (MPA) descrito na Equação (5) e analisou-se a magnitude dos erros para cada país. Para isso, definiu-se como $d$ a diferença entre o EQMP do MPA e o EQMP do MCC. Para de < que 0, considera-se que o MPA tem erro menor e infere-se que sua previsão é mais acurada para a amostra em questão. Para $d>0$, infere-se que o MCC apresenta mais acurácia em sua previsão.

A análise foi segregada para as observações dentro e fora da amostra de modo a detalhar a eficiência do modelo quanto à sua real capacidade de previsão. Para os dados dentro da amostra, os resultados apresentados na Tabela 4 mostram que para todos os países obteve-se um valor para $\mathrm{d}>0$, indicando que o modelo proposto (MCC) apresenta maior acurácia que um passeio aleatório (MPA). Isso significa que a taxa de câmbio contém informações relevantes para a previsão do índice de preços de commodities. 
Tabela 4 - Análise comparativa EQMP (MCC X MPA) - Dentro da Amostra

\begin{tabular}{lcccccc}
\hline & AUS & BR & CAN & CHI & COL & NZ \\
\hline MPA EQMP (1) & 0,00119 & 0,00265 & 0,00315 & 0,00539 & 0,00406 & 0,00085 \\
MCC EQMP (2) & 0,00109 & 0,00253 & 0,00310 & 0,00444 & 0,00375 & 0,00076 \\
$d(1-2)$ & 0,00009 & 0,00012 & 0,00005 & 0,00095 & 0,00030 & 0,00009 \\
\hline
\end{tabular}

Nota: AUS, Austrália; BR, Brasil; CAN, Canadá; CHI, Chile; COL, Colômbia; NZ, Nova Zelândia.

Para o período fora da amostra, os resultados apresentados na Tabela 5 também sugerem que variações na taxa de câmbio contêm informações relevantes para a previsão do índice de preços de commodities. Verifica-se que para todos os países, exceto a Colômbia, o EQMP do modelo proposto é menor do que o EQMP de um passeio aleatório $(d>0)$.

Tabela 5 - Análise comparativa EQMP (MCC X MPA) - Fora da Amostra

\begin{tabular}{lcccccc}
\hline & AUS & BR & CAN & CHI & COL & NZ \\
\hline MPA EQMP (1) & 0,00216 & 0,00262 & 0,00076 & 0,00209 & 0,00181 & 0,00096 \\
MCC EQMP (2) & 0,00167 & 0,00246 & 0,00066 & 0,00188 & 0,00230 & 0,00090 \\
$d(1-2)$ & 0,00049 & 0,00016 & 0,00011 & 0,00022 & $-0,00049$ & 0,00007 \\
\hline
\end{tabular}

Nota: AUS, Austrália; BR, Brasil; CAN, Canadá; CHI, Chile; COL, Colômbia; NZ, Nova Zelândia.

Além de analisar a magnitude da diferença do EQMP calculado para cada modelo, testou-se a significância estatística desta diferença utilizando-se o teste de igualdade de acurácia de previsões proposto por Diebold e Mariano (1995). Esse teste tem por objetivo avaliar comparativamente as previsões de dois modelos distintos através do diferencial de perda de cada previsão sob a hipótese nula de igual acurácia entre os modelos avaliados. Os resultados para o teste realizado no período dentro da amostra são apresentados na Tabela 6 .

Observa-se a significância estatística das inferências feitas a partir da comparação entre o EQMP dos modelos estudados. A rejeição da hipótese nula ${ }^{14}$ de que a acurácia dos modelos é igual, juntamente com o EQMP observado menor no MCC comparado ao MPA, mostram a superioridade do modelo proposto frente ao modelo de referência.

\footnotetext{
14 Para rejeitar a hipótese nula, é necessário um nível de significância de 10\% no caso do Brasil, Canadá e Chile.
} 
Logo, para este período da análise, é possível afirmar que a variação da taxa de câmbio contribui positivamente na previsão da variação do preço das commodities, uma vez que o MCC apresentou previsão consistentemente mais acurada que o MPA.

Tabela 6 - Análise comparativa Teste Diebold-Mariano (MCC X MPA) - Dentro da Amostra

\begin{tabular}{lcccccc}
\hline & AUS & BR & CAN & CHI & COL & NZ \\
\hline Estatística Diebold-Mariano (S) & 1,828 & 1,326 & 1,381 & 1,488 & 1,804 & 2,101 \\
P-valor Diebold-Mariano & $0,034^{* *}$ & $0,092^{*}$ & $0,084^{*}$ & $0,068^{*}$ & $0,036^{* *}$ & $0,018^{* *}$ \\
\hline
\end{tabular}

Nota: AUS, Austrália; BR, Brasil; CAN, Canadá; CHI, Chile; COL, Colômbia; NZ, Nova Zelândia.

* Rejeita-se H0 com nível de significância de 10\%, indicando evidência de diferença na acurácia das previões.

**Rejeita-se H0 com nível de significância de 5\%, indicando evidência de diferença na acurácia das previões.

A mesma análise foi estendida para o período fora da amostra e os resultados são apresentados na Tabela 7 . Neste caso, os resultados diferem daqueles encontrados dentro da amostra. Nota-se que a hipótese nula de igual acurácia nas previsões é rejeitada para o Canadá e para a Austrália. Para os demais países, não foi possível rejeitar a hipótese nula, indicando que as diferenças nas previsões não são significativas, apesar de se verificar anteriormente menores erros do MCC comparado ao MPA para todos os países, com exceção da Colômbia.

Tabela 7 - Análise comparativa Teste Diebold-Mariano (MCC X MPA) - Dentro da Amostra

\begin{tabular}{lcccccc}
\hline & AUS & BR & CAN & CHI & COL & NZ \\
\hline Estatística Diebold-Mariano (S) & 1,564 & 1,209 & 1,781 & 0,367 & $-1,736$ & 0,514 \\
P-valor Diebold-Mariano & $0,059^{\star}$ & 0,113 & $0,037^{\star *}$ & 0,357 & 0,959 & 0,304 \\
\hline
\end{tabular}

Nota: AUS, Austrália; BR, Brasil; CAN, Canadá; CHI, Chile; COL, Colômbia; NZ, Nova Zelândia.

* Rejeita-se H0 com nível de significância de 10\%, indicando evidência de diferença na acurácia das previões.

** Rejeita-se H0 com nível de significância de 5\%, indicando evidência de diferença na acurácia das previões.

Portanto, para o período considerado fora da amostra não é possível afirmar que a variação da taxa de câmbio contribui positivamente na previsão da variação do preço das commodities, com base no modelo estimado neste trabalho. 
É possível que o pequeno número de observações considerado para a análise fora da amostra tenha influenciado os resultados do teste estatístico, sendo possível que uma previsão com um maior número de observações para esse período resultasse em um melhor desempenho no teste estatístico.

\section{Conclusões}

Este trabalho averigua a relação dinâmica entre a variação dos preços das commodities e das taxas de câmbio na tentativa de constatar a existência de causalidade entre essas variáveis e as implicações que esta causalidade pode ter para fins de criação de modelos preditivos. Após selecionar uma amostra com países em que as commodities têm um peso relevante na pauta de exportações, identificou-se uma relação robusta de causalidade na qual flutuações na taxa de câmbio antecedem movimentos nos preços das commodities para Austrália, Brasil, Canadá, Chile, Colômbia e Nova Zelândia.

Para o período compreendido dentro da amostra, é possível afirmar que a taxa de câmbio é uma variável útil para a previsão dos preços das commodities, podendo ser usada com esta finalidade na composição de modelos econométricos. Entretanto, este resultado não se manteve para as observações consideradas fora da amostra, o que torna a análise ainda pouco conclusiva quanto à utilidade do modelo proposto para aplicações práticas.

Para trabalhos futuros, um possível aprimoramento seria a utilização de uma cesta de moedas dos principais países exportadores de commodities, bem como um índice global de preços de commodities. 


\section{Referências}

CASHIN, P., Céspede, L. F. e Sahay, R. Commodity Currencies and the Real Exchange Rate. Journal of Development Economics 75, 239-268, 2004.

CHEN, Y., Rogoff, K. e Rossi, B. Can Exchange Rates Forecast Commodity Prices? The Quarterly Journal of Economics 125 (3), 1145-1194, 2010.

CLEMENTS, M. P. e Hendry, D. F. Forecasting Economic Time Series. Cambrige: Cambrige University Press, 1998.

DIEBOLD, F. e Mariano, R. Comparing Predictive Accuracy. Journal of Business and Economic Statistics 13, 253-265, 1995.

ENGEL, C. e West, K. D. Exchange Rates and Fundamentals. Journal of Political Economy 113 (3), 485-517, 2005.

MEESE, R. A. e Rogoff, K. Empirical Exchange Rate Models of the Seventies. Do They Fit Out of Sample? The Journal of International Economics 14, 3-24, 1983.

PINDYCK, R. S. e Rotemberg, J. J. The Excess Co-movement of Commodity Prices. The Economic Journal 100, 1173-1189, 1990.

SARNO, L. e Valente, G. Exchange Rates and Fundamentals: Footloose or Evolving Relationship. Journal of the European Economic Association 7, 786-830, 2009.

VERÍSSIMO, M. P., Xavier, L. C. e Vieira, J. J. Taxa de Câmbio e Preços de Commodities: Uma Investigação sobre a Hipótese da Doença Holandesa no Brasil. Anpec Vol. 13(1), 2012. 